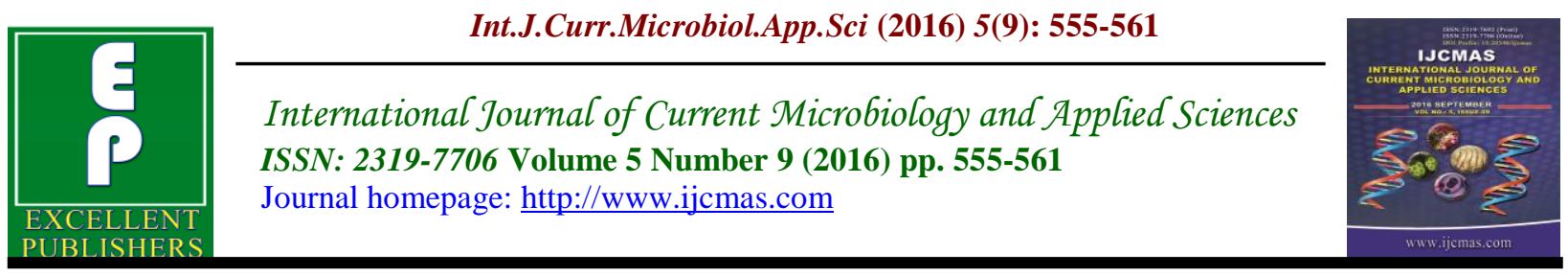

Original Research Article

http://dx.doi.org/10.20546/ijcmas.2016.509.062

\title{
Investigation of Bacterial Infections in Pig Farms affected by Respiratory Disease Complex in Bulgaria
}

\author{
Roman Pepovich $^{1}$, Branimir Nikolov ${ }^{2}$, Kalin Hristov ${ }^{3}$, Krasimira Genova ${ }^{4}$, \\ Radka Hadjiolova-Tafradjiyska ${ }^{5}$ and Stanislav Radanski ${ }^{1}$
}

${ }^{1}$ Department of Infectious Pathology and Hygiene, Technology and Control of Foods from

Animal Origin, Faculty of Veterinary Medicine, University of Forestry, Sofia, Bulgaria

${ }^{2}$ Department of Internal Diseases, Pathology and Pharmacology, Faculty of Veterinary Medicine, University of Forestry, Sofia, Bulgaria

${ }^{3}$ Department of Surgery, Radiology, Obstetrics and Gynecology, Faculty of Veterinary Medicine, University of Forestry, Sofia, Bulgaria

${ }^{4}$ Department of Animal Breeding Science, Faculty of Veterinary Medicine, University of

Forestry, Sofia, Bulgaria

${ }^{5}$ Department of Pathophysiology, Faculty of Medicine, Medical University, Sofia, Bulgaria

*Corresponding author

\section{A B S T R A C T}

\section{Keywords}

Pigs, respiratory disease complex, bacterial infections, antimicrobial susceptibility.

\section{Article Info}

Accepted:

20 August 2016

Available Online:

10 September 2016
This study attempted to identify bacterial pathogens and determine their importance in porcine respiratory disease complex in order to identify effective and adequate measures for their control. The study was conducted in three industrial intensive pig farms in different regions of Bulgaria. Nasal swab samples from weaners and fattening pigs affected by respiratory infection were examined, as well as from the lungs of animals that had died or had been emergency slaughtered, with changes characteristic of pneumonia. The results showed that mono-infection was prevalent in the analyzed nasal samples in weaners - $54.2 \%$, and in $70.8 \%$ alpha hemolytic Streptococcus was detected. In fattening pigs, associated infections dominated with $54.2 \%$. In a significant part of the samples $(62.5 \%)$ there were observed alpha hemolytic Streptococcus and S. epidermidis. The results from the microbiological examinations of the lungs showed that the prevailing cases were mono-infections in weaners pigs (70.8\%), as well as in fattening pigs (75.0\%). A significant proportion of lung specimens were shown to contain $E$. coli, probably due to coli septicemia potentiated by $M$. hyopneumoniae.

\section{Introduction}

Respiratory diseases remain a current problem in industrial pig farming, regardless of the herd size and the rearing technologies. These diseases very often occur with multiple etiology, which has historically led to the coining of the term Porcine respiratory disease complex (PRDC) (Motovski, 2003; Bochev, 2007). 
The PRDC etiology varies considerably in different production systems (Halbur, 1999; Motovski, 2003). It commonly involves one or two viruses, Mycoplasma hyopneumoniae (M. hyopneumoniae, M. hyo) and other bacterial agents acting together to cause severe respiratory diseases and, consequently, major economic losses (Motovski, 2003).

One of the major etiological agents of PRDC is M. hyopneumoniae, the causative agent of enzootic pneumonia in pigs (Stipkovits et al., 2001; Opriessnig et al., 2004; Thacker, 2006). M. hyopneumoniae infection in pigs is a predisposing factor for the development of secondary bacterial infections of the respiratory tract (Bosch, 2004). According to Tielen (1995), some of the most common secondary causative agents include Pasteurella multocida ( $P$. m.), Streptococcus suis (S. suis), Staphylococcus aureus (S. aureus) etc.

The aim of this study was to identify the bacterial causative agents and determine their role in PRDC in three different pig farms in Bulgaria in order to define effective and adequate control measures.

\section{Materials and Methods}

\section{Samples}

Samples were collected in 2014 from three pig farms located in different parts of Bulgaria with incidence of respiratory infections. The studied clinical samples included 48 nasal swabs and 48 lung samples from growing and fattening pigs.

Nasal swabs were taken by deep rubbing of dry sterile swabs against the nasal mucosa on one side. Each swab was then inserted into a container with transport medium $\left(\mathrm{COPAN}^{\circledR}\right)$. Lung samples were taken from dead growing and fattening pigs and from emergency slaughtered pigs with pneumotic changes. Each collected sample was inserted into a sterile container.

\section{Microbiological analysis}

The nasal swabs and lung samples were enriched in standard broth containing $1 \%$ dextrose (BulBio-NCIPD). Then plating was done on conventional nutrient media for primary isolation of bacterial respiratory pathogens: blood agar $\left(\right.$ BioMerieux $\left.^{\circledR}\right)$; MacConkey agar (BioMerieux ${ }^{\circledR}$ ); Chocolate agar with IsoVitalex and Bacitracin (Becton, Dickinson and Company $\left.{ }^{\circledR}\right)$; deoxycholate citrate lactose agar (BulBio ${ }^{\circledR}$ - NCIPD); Kligler agar $\quad$ BulBio $^{\circledR}{ }^{\circledR}$ NCIPD). Identification of the obtained isolates was done according to the classical procedures for routine microbial diagnostics and by using automated methods for identification of bacteria (Gram-staining; colony morphology; catalase test with $3 \% \quad \mathrm{H}_{2} \mathrm{O}_{2}$; $\mathrm{H}_{2} \mathrm{~S}$ production; latex slide agglutination test for Staphylococcus aureus and Coagulasenegative staphylococci (STAPHYTECT PLUS, Oxoid ${ }^{\circledR}$ ); latex slide agglutination test for beta-hemolytic Streptococcus (SLIDEX Strepto Plus, BioMérieux ${ }^{\circledR}$ ) and a VITEK 2 compakt (BioMerieux ${ }^{\circledR}$ ) automated instrument.

To determine the antibiotic sensitivity of the isolates, antibiograms were obtained by the disc diffusion assay, using the following nutrient media: Mueller Hinton 2 agar and Mueller Hinton 2 agar $+5 \%$ sheep blood $\left(\right.$ BioMerieux $^{\circledR}$ ), and BD BB Sensi-Disc Antimicrobial Susceptibility Test Discs (Becton Dickinson and Company ${ }^{\circledR}$ ) (Table 1).

\section{Serological tests}

The etiological role of $M$. hyopneumoniae and $A$. pleuropneumoniae in respiratory 
diseases in pigs was diagnosed using enzyme immunoassays for detection of specific antibodies in blood sera of growing and fattening pigs from the studied pig farms.

Blocking ELISA was used for detection of specific antibodies against $M$. hyopneumoniae (Ingezim M. hyo compact, INGENASA $^{\circledR}$ ) and indirect ELISA, for detection of specific antibodies against $A$. pleuropneumoniae (Ingezim APP mix, INGENASA $^{\circledR}$ ). All procedures were strictly based on the manufacturer's instructions.

\section{Results and Discussion}

The data from the microbiological analysis of the nasal swabs from growing and fattening pigs originating from pig farms with respiratory symptoms are presented in Fig. 1 and Fig. 2. The obtained results showed that, in the studied farms, monoinfection was predominant in the growing pigs $(54.2 \%)$. The majority of the isolates were identified as alpha-hemolytic Streptococcus $(70.8 \%)$. There was also high prevalence of infections caused by $S$. epidermidis (37.5\%). In $12.5 \%$ of the cases, E. coli strains were identified. Klebsiella spp. and $S$. aureus had similar prevalence of $8.3 \%$. In the fattening pigs, there were predominantly associated infections $(54.2 \%)$. In this group of pigs, the microbiological tests did not reveal pathogenic $E$. coli and $S$. aureus in the three farms examined by us. The most prevalent pathogens in the fattening pigs were identified to be alpha hemolytic Streptococcus and S. epidermidis, both with $62.5 \%$ prevalence. In $33.3 \%$ of the cases, Klebsiella spp. were isolated.

The microbiological analysis of the lung samples taken from dead pigs with clinical signs of respiratory disease (Fig. 3 and Fig. 4) showed that the infections that prevailed in the growing pigs were mono-infections $(70.8 \%)$. Most of the isolates were identified as E. coli; then Klebsiella spp., S. aureus and Coagulase-negative staphylococci $(C N S)$ ranked second, with $8.3 \%$ each. There was a comparatively low prevalence of alpha hemolytic Streptococcus (4.2\%).

Table.1 Antibacterial discs used

\begin{tabular}{|l|l|c|c|}
\hline \multicolumn{1}{|c|}{ Antibiotic group } & \multicolumn{1}{c|}{ Antimicrobial disc } & Code & $\boldsymbol{\mu g} / \mathbf{d i s c}$ \\
\hline 1. Aminopenicillines & Amoxicillin & AMX & 25 \\
\hline \multirow{2}{*}{ 2. Cephalosporines } & Cephalexin & $\mathrm{CN}$ & 30 \\
\hline \multirow{2}{*}{ 3. Aminosides } & Gentamicin & $\mathrm{GM}$ & 10 \\
\cline { 2 - 4 } & Amikacin & $\mathrm{AN}$ & 30 \\
\hline 4. Tetracyclines & Tetracyclin & $\mathrm{TE}$ & 30 \\
\hline 5. Quinolones & Ciprofloxacin & $\mathrm{CIP}$ & 5 \\
\hline 6. Sulphonamides & Trimethoprim/Sulfamethoxazole & SXT & $1,25-23,75$ \\
\hline
\end{tabular}


Int.J.Curr.Microbiol.App.Sci (2016) 5(9): 555-561

Table.2 Results of serological tests for proof of specific antibodies against M. hyopneumoniae and A. pleuropneumoniae

\begin{tabular}{|c|c|c|c|c|}
\hline \multirow{3}{*}{ swine farms } & \multicolumn{2}{|c|}{ Antibody against } & \multicolumn{2}{c|}{ Antibody against } \\
\cline { 2 - 5 } & \multicolumn{2}{|c|}{ M. hyopneumoniae } & \multicolumn{2}{c|}{ A. pleuropneumoniae } \\
\cline { 2 - 5 } & growing pigs & fattening pigs & growing pigs & fattening pigs \\
\hline 1 & + & + & - & + \\
\hline 2 & + & + & - & + \\
\hline 3 & + & + & + & + \\
\hline
\end{tabular}

Table.3 Sensitivity of microorganisms isolated from nasal swab samples from growing and fattening pigs to certain antibiotic agents

\begin{tabular}{|c|c|c|c|c|c|c|c|c|c|c|c|c|c|c|c|}
\hline \multirow{3}{*}{ Antibiotic } & \multicolumn{3}{|c|}{ E. coli } & \multirow{2}{*}{\multicolumn{3}{|c|}{$\begin{array}{c}\text { Klebsiella spp. } \\
(n=10)\end{array}$}} & \multicolumn{3}{|c|}{ S. aureus } & \multicolumn{3}{|c|}{$C N S$} & \multicolumn{3}{|c|}{$\alpha$-Strept. } \\
\hline & & $l=$ & & & & & & $=$ & & & $=2$ & & & $=3$ & \\
\hline & $R$ & $I$ & $S$ & $R$ & $I$ & $S$ & $R$ & $I$ & $S$ & $R$ & $I$ & $S$ & $R$ & $I$ & $S$ \\
\hline AMX $-25 \mu \mathrm{g}$ & 3 & - & - & 7 & 1 & 2 & - & 1 & 1 & 2 & 5 & 17 & - & 8 & 24 \\
\hline $\mathrm{CN}-30 \mu \mathrm{g}$ & - & 2 & 1 & 3 & - & 7 & - & - & 2 & - & 2 & 22 & - & 2 & 30 \\
\hline GM - $10 \mu \mathrm{g}$ & - & - & 3 & - & - & 10 & - & - & 2 & - & 1 & 23 & - & 5 & 27 \\
\hline $\mathrm{AN}-30 \mu \mathrm{g}$ & - & - & 3 & - & - & 10 & - & - & 2 & 17 & 4 & 3 & 22 & 8 & 2 \\
\hline $\mathrm{TE}-30 \mu \mathrm{g}$ & 3 & - & - & 4 & 1 & 5 & 2 & - & - & 21 & 1 & 2 & 30 & 2 & - \\
\hline CIP $-5 \mu \mathrm{g}$ & - & - & 3 & - & - & 10 & - & - & 2 & 7 & - & 17 & 5 & 4 & 23 \\
\hline SXT $-1.25-23,75 \mu \mathrm{g}$ & 3 & - & - & 3 & 3 & 4 & - & 1 & 1 & 9 & 3 & 12 & 15 & 2 & 15 \\
\hline
\end{tabular}

Table.4 Sensitivity of microorganisms isolated from lungs of dead and slaughtered growing and fattening pigs to certain antibiotic agents

\begin{tabular}{|c|c|c|c|c|c|c|c|c|c|c|c|c|c|c|c|}
\hline \multirow{3}{*}{ Antibiotic } & \multicolumn{3}{|c|}{ E. coli } & \multicolumn{3}{|c|}{ Klebsiella spp. } & \multicolumn{3}{|c|}{ S. aureus } & \multicolumn{3}{|c|}{$C N S$} & \multicolumn{3}{|c|}{$\alpha$-Strept. } \\
\hline & & $t=4$ & & & $n=2$ & & & $n=4$ & & & $=1$ & & & $=$ & \\
\hline & $R$ & $I$ & $S$ & $R$ & $I$ & $S$ & $R$ & $I$ & $S$ & $R$ & $I$ & $S$ & $R$ & $I$ & $S$ \\
\hline AMX - $25 \mu \mathrm{g}$ & 26 & 1 & 13 & 2 & - & - & - & 2 & 2 & - & 1 & 9 & - & - & 2 \\
\hline $\mathrm{CN}-30 \mu \mathrm{g}$ & 4 & 13 & 23 & 2 & - & - & - & - & 4 & - & - & 10 & - & - & 2 \\
\hline GM - $10 \mu \mathrm{g}$ & - & 4 & 36 & 2 & - & - & - & - & 4 & - & - & 10 & - & - & 2 \\
\hline $\mathrm{AN}-30 \mu \mathrm{g}$ & - & 1 & 39 & - & - & 2 & - & - & 4 & - & 3 & 7 & 2 & - & - \\
\hline $\mathrm{TE}-30 \mu \mathrm{g}$ & 39 & - & 1 & 2 & - & - & 4 & - & - & 8 & 2 & - & 2 & - & - \\
\hline CIP $-5 \mu \mathrm{g}$ & 6 & 1 & 33 & - & - & 2 & 1 & 2 & 1 & - & - & 10 & - & - & 2 \\
\hline SXT - 1.25-23,75 $\mu \mathrm{g}$ & 17 & - & 23 & 2 & - & - & - & 2 & 2 & - & 1 & 9 & - & - & 2 \\
\hline
\end{tabular}


Fig.1 Percent of pigs in growing and fattening affected by mono infection and associated infections (nasal samples)

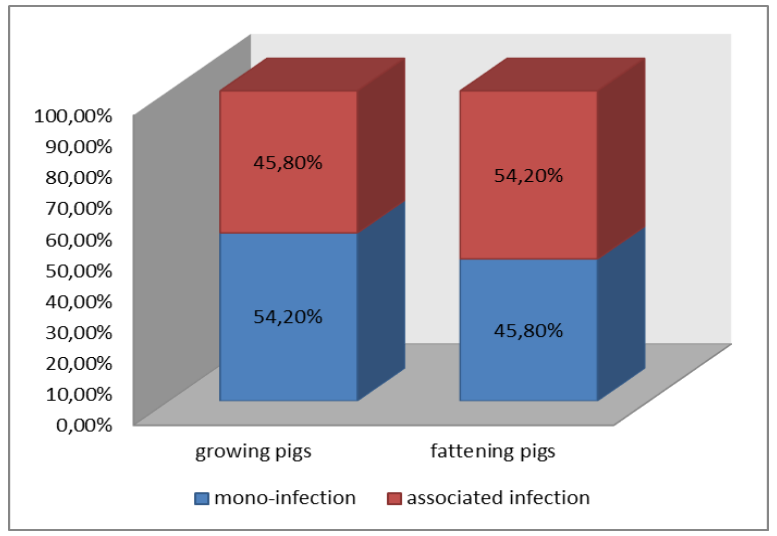

Fig.2 Results of microbiological studies on nasal swab samples from growing and fattening pigs (\%)

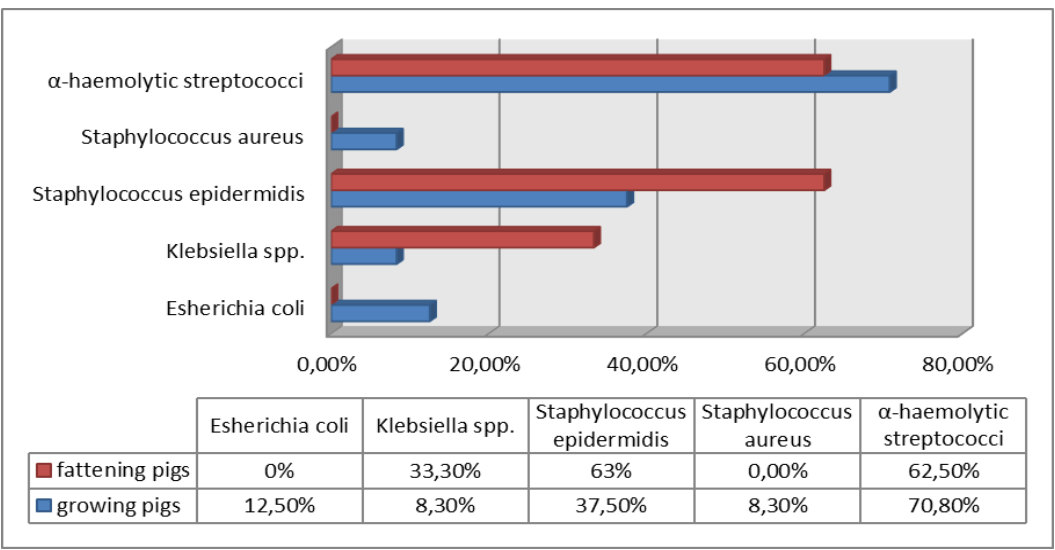

Fig.3 Percent of pigs in growing and fattening affected by mono infection and associated infections (lung samples)

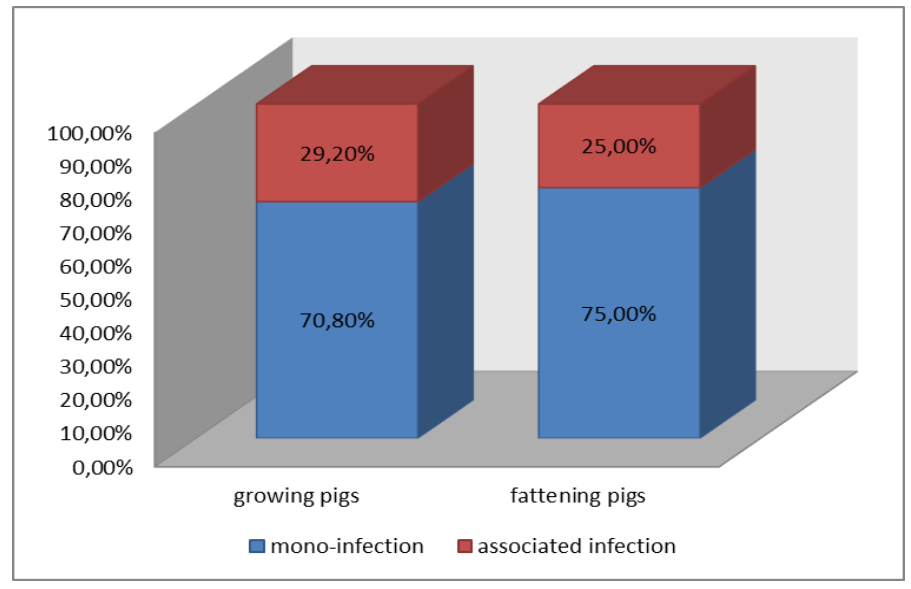


Fig.4 Results of microbiological studies on lungs from growing and fattening pigs (\%)

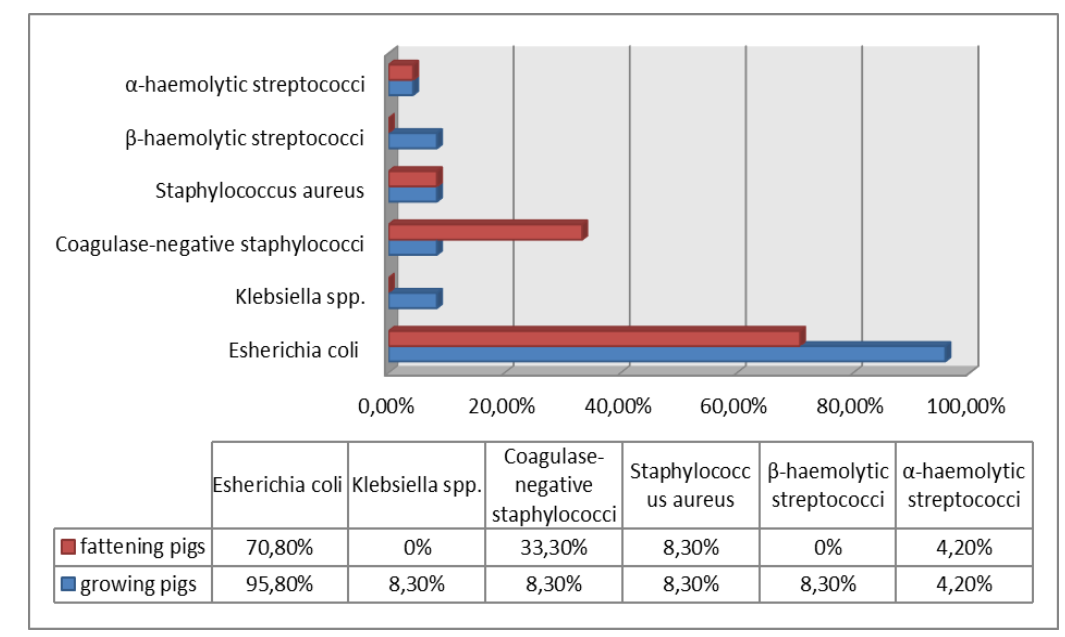

Interestingly, beta hemolytic Streptococcus was isolated in one of the studied farms. In the fattening pigs, the dominant infections were also mono-infections (75.0\%), with $E$. coli being the leading causative agent, and CNS ranking second. All the tested lung samples were negative for Klebsiella spp. and beta hemolytic Streptococcus. Other etiological agents that were isolated included S. aureus and alpha hemolytic Streptococcus, in $8.3 \%$ and $4.2 \%$ of the cases, respectively.

Our results concerning the detection of $M$. hyopneumoniae and A. pleuropneumoniae infections in the studied farms (Table 2) showed that antibodies against $M$. hyopneumoniae were found in different technological groups of pigs in all the three studied farms. In two of the farms, none of the tested samples from the growing pigs was positive for A. pleuropneumoniae. Conversely, among the fattening pigs, such antibodies were detected in all the three farms. In pigs, infection with $M$. hyopneumoniae and A. pleuropneumoniae leads to suppression of the phagocytic activity of alveolar macrophages, resulting in immunosuppression and thus predisposing the animals to secondary bacterial infections of the respiratory tract.
The patterns of in vitro antibiotic sensitivity of the microbial isolates from the nasal swabs of growing and fattening pigs are presented in Table 3. The obtained results showed that the E. coli isolates were resistant to amoxicillin, tetracycline and sulfametoxazol+trimetoprim and susceptible to gentamicin, amikacin and ciprofloxacin. Similar patterns of antibiotic sensitivity to gentamicin, amikacin and ciprofloxacin were observed in all Klebsiella spp. isolates. Most strains showed amoxicillin resistance. S. aureus demonstrated good sensitivity to cephalexin, gentamicin, amikacin and ciprofloxacin, but was absolutely resistant to tetracycline. The antimicrobial activity of amoxicillin, cephalexin, gentamicin and ciprofloxacin against most alpha hemolytic Streptococcus isolates was good, but that of amikacin and tetracycline was poor.

The response of the microbial isolates from the lung samples of the growing and fattening pigs to different antimicrobial agents are shown in Table 4. The majority of the $E$. coli isolates from lung samples had good sensitivity to gentamicin, amikacin and ciprofloxacin, but were resistant to amoxicillin and tetracycline. All the tested Klebsiella spp. isolates showed resistance to most of the antibiotic agents included in this 
study, except amikacin and ciprofloxacin. In our study, $S$. aureus was observed to have good sensitivity to cephalexin, gentamicin and amikacin and resistance to tetracycline. A lot of CNS isolates exhibited sensitivity to the tested antimicrobial agents, except tetracycline. Our data indicate that the prevailing type of antibiotic resistance among all the tested alpha hemolytic Streptococcus strains was that to amikacin and tetracycline, whereas the sensitivity to the other antimicrobial agents included in this study was preserved.

In conclusion, the prevalent pathogen in the majority of the studied nasal swabs from growing and fattening pigs with clinical signs of respiratory infection, was alpha hemolytic Streptococcus. The prevalent infections in the affected lungs of weaning and fattening pigs were shown to be monoinfections, with $E$. coli as the leading cause. The majority of the Gram-positive and Gram-negative bacterial isolates showed high levels of resistance against tetracycline antibiotics. $M$. hyopneumoniae and $A$. pleuropneumoniae were demonstrated to play a role in the multiple etiology of respiratory diseases in pigs.

\section{References}

Bochev, I. 2007. Porcine respiratory disease complex (PRDC): A review. I. Etiology, epidemiology, clinical forms and pathoanatomical features. Bulg. $J$. Vet. Med., 10: 131-146.
Bosch, G. 2004. Single-dose treatment against respiratory bacteria. Pig international. 34, 30-32.

Halbur, P.G. 1999. Porcine respiratory disease complex. Proc. of the North Carolina Healthy Hogs Seminar (North Carolina Swine Veterinary group), 1-14.

Motovski, A. 2003. Respiratoren bolesten kompleks. Veterinarnomeditsinski novini, 3(4): 24-28.

Opriessnig, T., Thacker, E.L., Yu, S., Fenaux, M., Meng, X.J. and Halbur, P.G. 2004. Experimental reproduction of postweaning multisystemic wasting syndrome in pigs by dual infection with Mycoplasma hyopneumoniae and porcine circovirus type 2. Vet. Pathol., 41: 624-640.

Stipkovits, L., Miller, D., Glavits, R., Fodor, L. and Burch, D. 2001. Treatment of pigs experimentally infected with Mycoplasma hyopneumoniae, Pasteurella multocida, and Actinobacillus pleuropneumoniae with various antibiotics. Can. J. Vet. Rec., 65: 213-222.

Thacker, E.L. 2006. Mycoplasmal diseases. In: Leman, A.D., Straw, B.E., D'Allaire, S., Mengeling, W.L., and Taylor, D.J., (Ed.), Diseases of Swine, 9th ed. The Iowa State University Press, Ames, IA, 701-717.

Tielen, M. 1995. Respiratory diseases in pigs. Prevalence and economical effects. Pigs, Special, Resp. Dis., 4-5.

\section{How to cite this article:}

Roman Pepovich, Branimir Nikolov, Kalin Hristov, Krasimira Genova, Radka HadjiolovaTafradjiyska and Stanislav Radanski. 2016. Investigation of Bacterial Infections in Pig Farms affected by Respiratory Disease Complex in Bulgaria. Int.J.Curr.Microbiol.App.Sci. 5(9): 555561. doi: http://dx.doi.org/10.20546/ijcmas.2016.509.062 